\title{
Vilnius Photometry in the Southern Hemisphere
}

\author{
R.J. Dodd ${ }^{1}$, M.C. Forbes ${ }^{2}$, D.J. Sullivan ${ }^{2}$ \\ ${ }^{1}$ Carter Observatory, P.O. Box 2909, Wellington, New Zealand. \\ ${ }^{2}$ Victoria University of Wellington, New Zealand.
}

\begin{abstract}
The seven intermediate passband filters of the Vilnius photometric system enable a more comprehensive and discriminating classification of stellar objects than is possible in broadband systems. To date, a lack of suitable standard stars has inhibited its use in the Southern Hemisphere. We have an established programme for extending the network of northern Vilnius standards into the southern skies. This paper briefly summarizes the characteristics of the Vilnius system, provides a synopsis of the data obtained on over 200 standard stars so far and presents preliminary $\mathrm{CM}$ and $\mathrm{CC}$ diagrams for the open cluster Omicron Velorum (IC2391).
\end{abstract}

\section{Introduction}

A programme of Vilnius intermediate band stellar photometry for the southern hemisphere was first mooted at the Royal Observatory Edinburgh in 1985. A suitable set of filters was kindly provided by Professor V. Straižys (Institute of Theoretical Physics and Astronomy, Vilnius, Lithuania) and observations begun by Sullivan and Dodd in 1988. The initial goal of the observing programme was to establish standards both near the South Celestial Pole and for selected bright stars $(V<7)$ across the entire sky south of $-20^{\circ}$. The rate of data gathering increased markedly when Forbes (Ph.D. research student) joined the group and to date (August 1992) some 109 Vilnius primary standards, 225 southern hemisphere secondary standards and 90 cluster stars have been observed at least once.

\section{Instrumentation and Observations}

The passbands of the New Zealand set of Vilnius filters are shown in figure 1 superimposed on the spectra for an early (A) and a late (M) type star, obtained from the spectrophotometric atlas of Gunn \& Stryker (1983). The rationale for using this particular set of filters is given in detail by Straižys (1992). The Vilnius photometry allows the determination of spectral type, luminosity class, chemical composition parameter and interstellar reddening.

Observations have been made using the $61 \mathrm{~cm}$ telescopes at the $\mathrm{Mt}$. John $\mathrm{Ob}$ servatory of the University of Canterbury near Lake Tekapo in New Zealand. The thermoelectrically cooled photometer uses an EMI 9558B tube operated at $1700 \mathrm{v}$. The most commonly used photometer aperture was 32 arcsec diameter. The form 


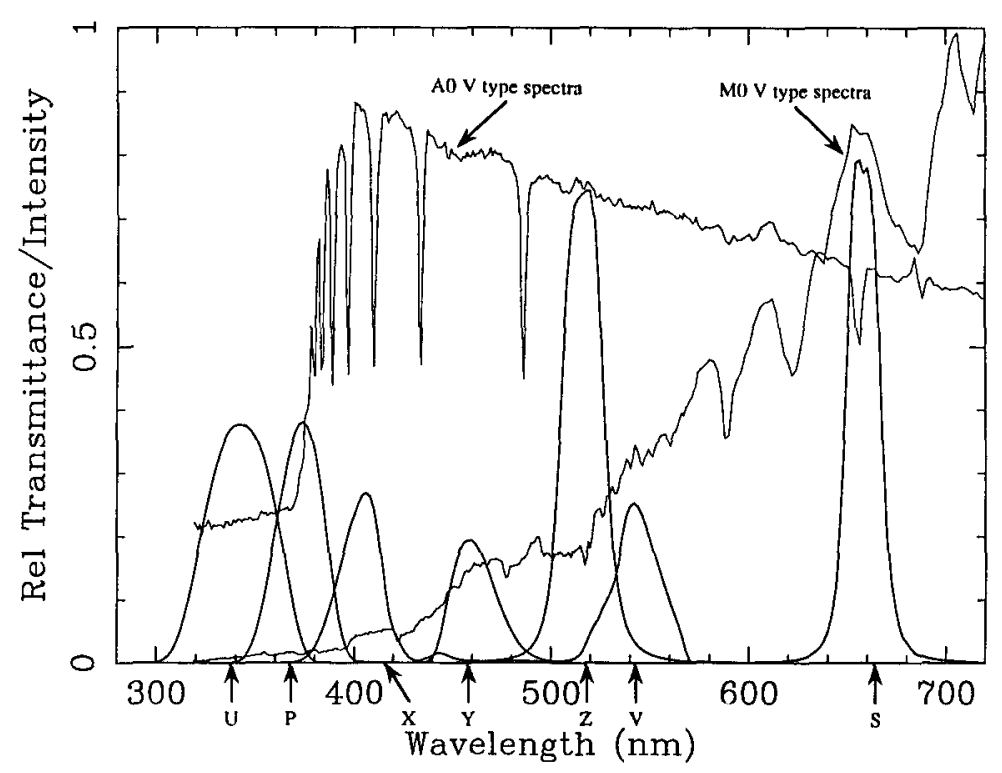

Figure 1: Southern Hemisphere Vilnius filter set passbands superimposed on an A and $M$ type stellar spectra.

Vilnius $\mathrm{X}$ extinction at Mt John

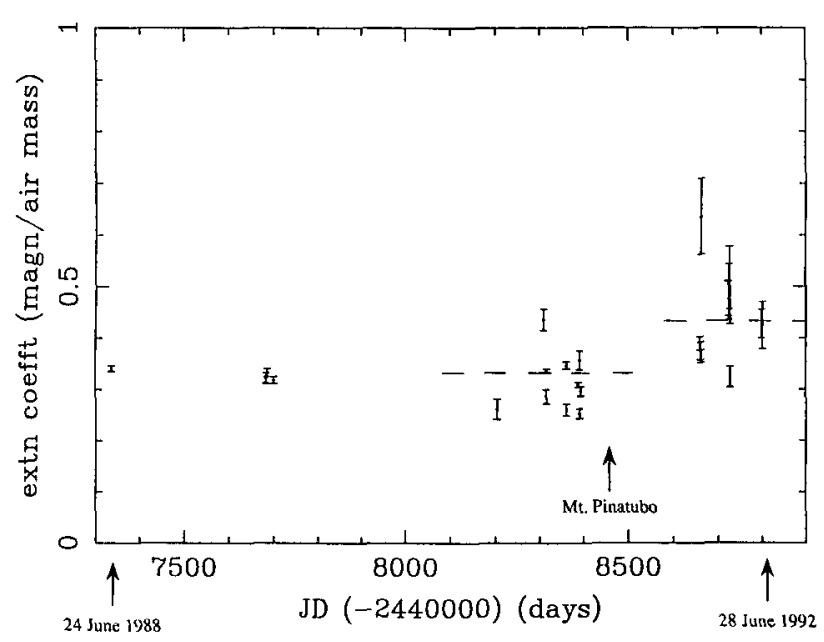

Figure 2: Vilnius X-band extinction at the Mt. John Observatory 1988-92. 
of observing followed that set out by Straižys (1992) so extinction and control stars were observed in addition to standard and programme stars. The filter observing sequence being :

(UPXYZVS)* check star centering (UPXYZVS)* (UPXYZVS) $)_{s k y}$.

Integration times varied from 5 to 100 seconds, with three integrations per colour for integrations up to 20 s and two integrations per colour for longer integrations. For the longer integrations, image centering was checked more frequently than every filter set run. The preliminary reduction of the observations presented in this paper used the method described by Hardie (1962). A more comprehensive reduction of the data is in progress, including removal of colour and time-dependent extinction effects.

To obtain a measure of the reliability of the southern standards and to tie the southern to the northern hemisphere standards, a series of observations of selected northern standards, 109 stars listed in the various Vilnius catalogues in all, was carried out at Mt. John. The relationships between southern $(S)$ and northern $(N)$ measurements of magnitude and colour for two typical nights (pre and post Mt. Pinatubo eruption) were as follows :

\begin{tabular}{|c|c|c|}
\hline 1989 June 8 & 1992 June 27 \\
\hline$V_{S}=(0.971 \pm 0.009) V_{N}-(1.075 \pm 0.062)$ & $V_{S}=(0.993 \pm 0.012) V_{N}$ & $(1.114 \pm 0.084)$ \\
$(U-P)_{S}=(1.037 \pm 0.027)(U-P)_{N}+(0.409 \pm 0.007)$ & $(U-P)_{S}=(0.840 \pm 0.141)(U-P)_{N}+(0.518 \pm 0.024)$ \\
$(P-X)_{S}=(0.963 \pm 0.020)(P-X)_{N}+(0.266 \pm 0.012)$ & $(P-X)_{S}=(0.936 \pm 0.050)(P-X)_{N}+(0.544 \pm 0.029)$ \\
$(X-Y)_{S}=(1.089 \pm 0.007)(X-Y)_{N}+(0.520 \pm 0.004)$ & $(X-Y)_{S}=(1.043 \pm 0.019)(X-Y)_{N}+(0.748 \pm 0.015)$ \\
$(Y-Z)_{S}=(0.847 \pm 0.012)(Y-Z)_{N}-(0.357 \pm 0.010)$ & $(Y-Z)_{S}=(0.769 \pm 0.025)(Y-Z)_{N}-(0.369 \pm 0.026)$ \\
$(Z-V)_{S}=(1.108 \pm 0.017)(Z-V)_{N}+(1.516 \pm 0.021)$ & $(Z-V)_{S}=(1.152 \pm 0.041)(Z-V)_{N}+(1.640 \pm 0.048)$ \\
$(V-S)_{S}=(0.886 \pm 0.011)(V-S)_{N}+(0.816 \pm 0.006)$ & $(V-S)_{S}=(0.868 \pm 0.015)(V-S)_{N}+(0.678 \pm 0.005)$ \\
\hline
\end{tabular}

Using these equations, which were determined from 12 standard stars, the standard deviations about the mean values of magnitude $\left(V_{N}-V_{S}\right)$ and colours $\left(C_{N}-C_{S}\right)$ were as follows :

\begin{tabular}{|lccccccc|}
\hline & $\sigma(\Delta V)$ & $\sigma(\Delta(U-P))$ & $\sigma(\Delta(P-X))$ & $\sigma(\Delta(X-Y))$ & $\sigma(\Delta(Y-Z))$ & $\sigma(\Delta(Z-V))$ & $\sigma(\Delta(V-S))$ \\
\hline 1989 June 8 & \pm 0.034 & \pm 0.028 & \pm 0.035 & \pm 0.019 & \pm 0.014 & \pm 0.009 & \pm 0.019 \\
1992 June 27 & \pm 0.039 & \pm 0.047 & \pm 0.046 & \pm 0.042 & \pm 0.019 & \pm 0.018 & \pm 0.016 \\
\hline
\end{tabular}

The standard deviations about the mean values of the magnitudes and colours in the Mt. John system only (i.e. internal comparison between measures of the same star made on the same night corrected for first order extinction) are typically smaller than $\pm 0^{m} \cdot 01$. The eruption of Mt. Pinatubo in the Philipines in June 1991 increased the average extinction at $\mathrm{Mt}$. John by some $0^{m} \cdot 1$ in the $\mathrm{X}$ passband (see figure 2). Also evident is the increased scatter in the measurements of atmospheric extinction after the eruption.

The presence of a colour term is well illustrated in figure 3 which shows the residuals in the $\mathrm{V}$ magnitude plotted against the composite ( $\mathrm{Y}-\mathrm{V})$ colour. The pairs of crosses are for the same standard star observed at different times. 


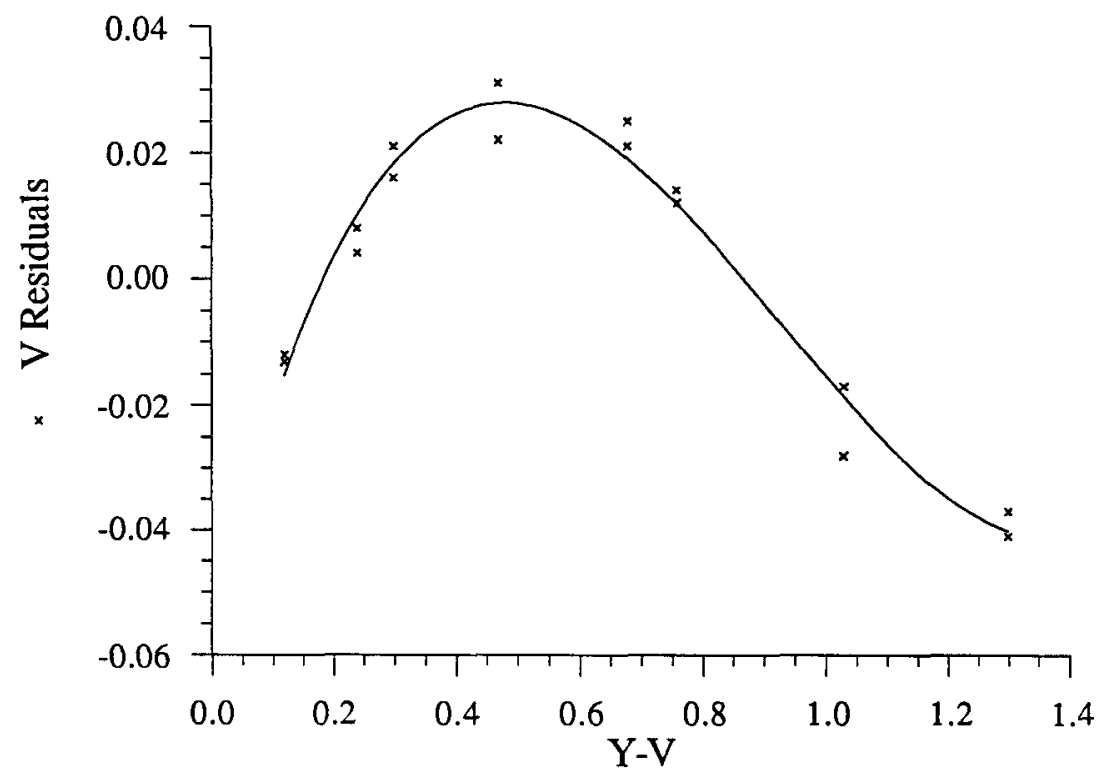

Figure 3: Colour dependence of standard star residuals 8 June 1989.

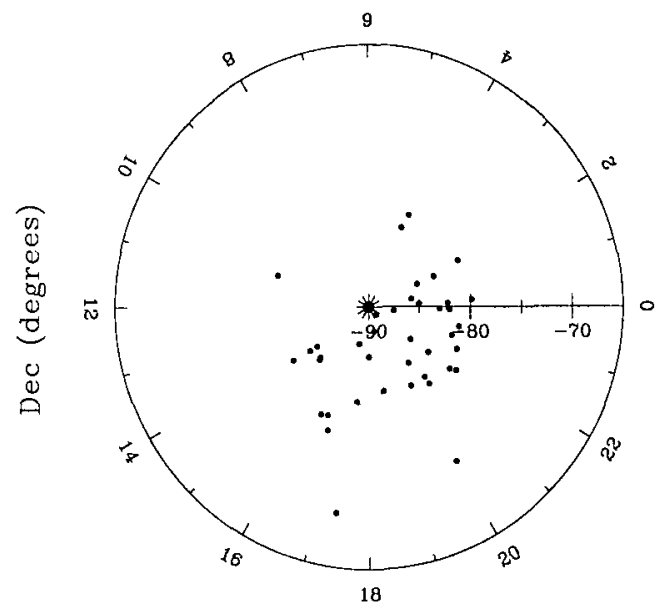

RA (Hours)

Figure 4: South celestial pole Vilnius secondary standards. 


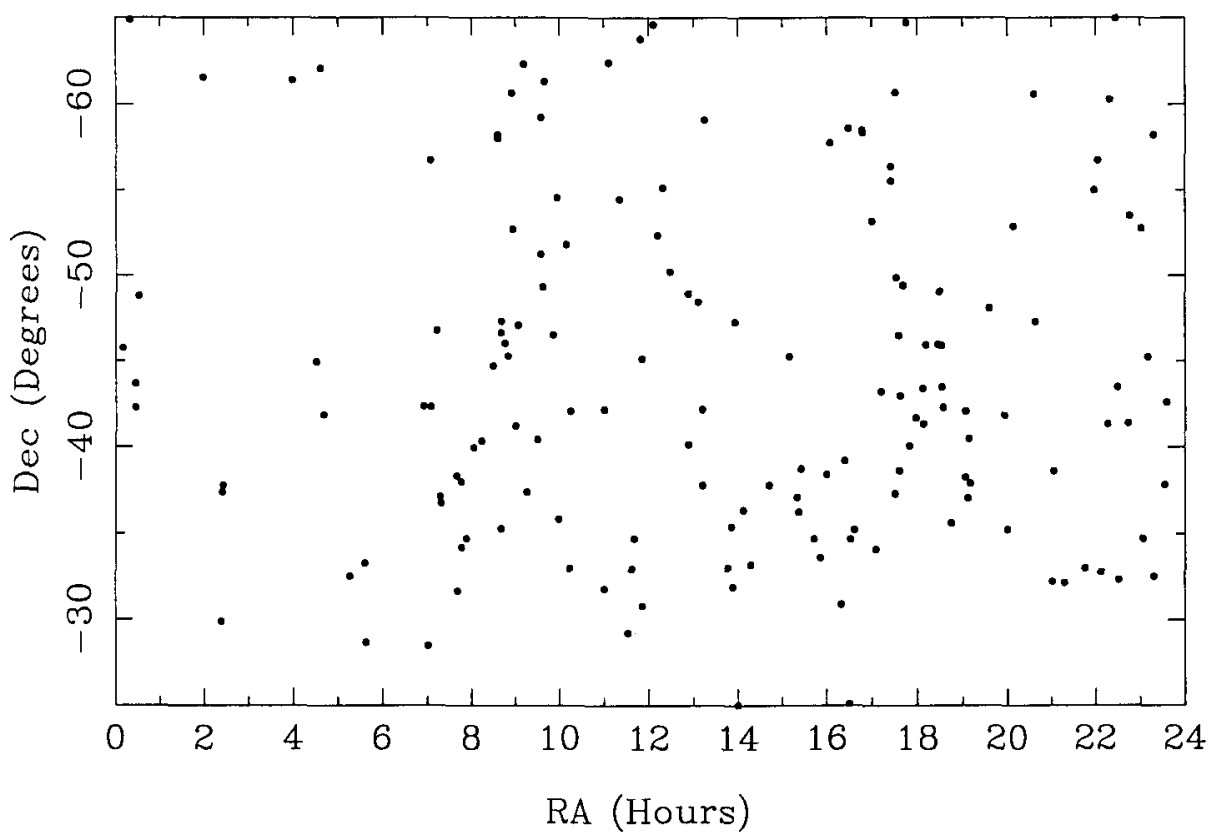

Figure 5: Vilnius secondary standards between $\delta=-20^{\circ}$ and $-70^{\circ}$.
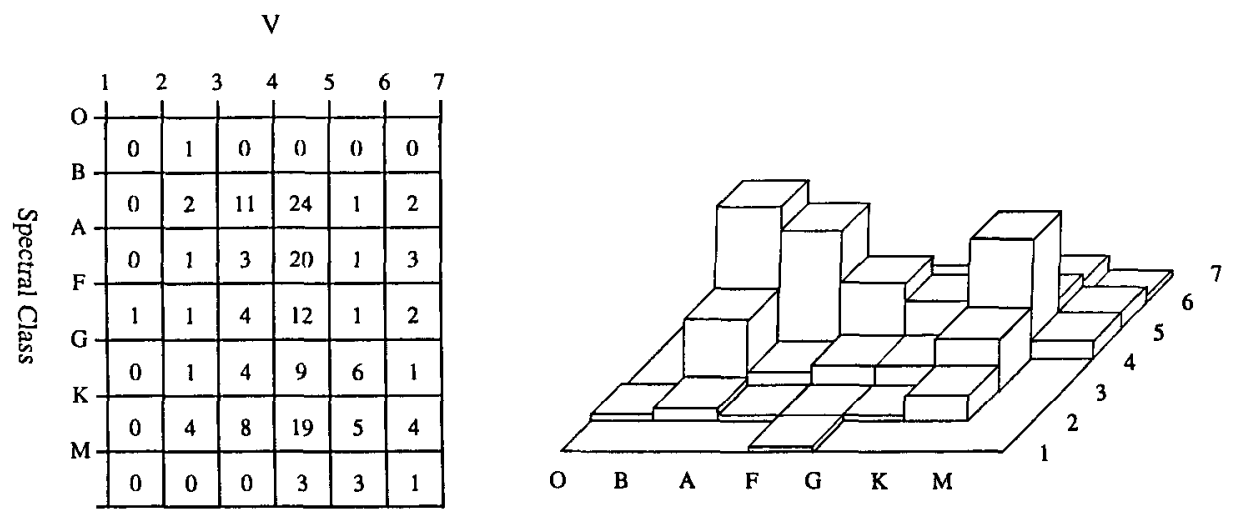

Figure 6: Magnitude - Spectral type histogram for Vilnius southern hemisphere secondary standards. 


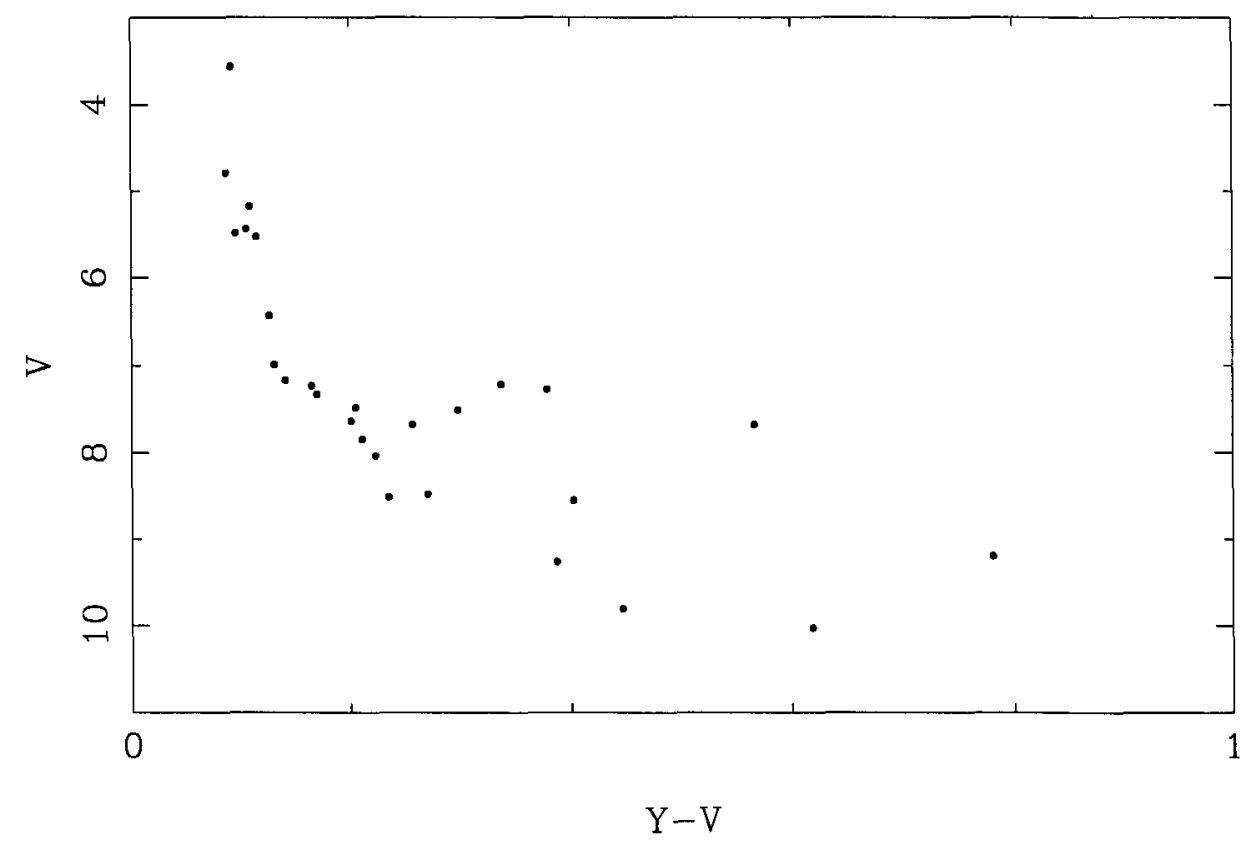

Figure 7: CMD for $O$ Vel galactic cluster.

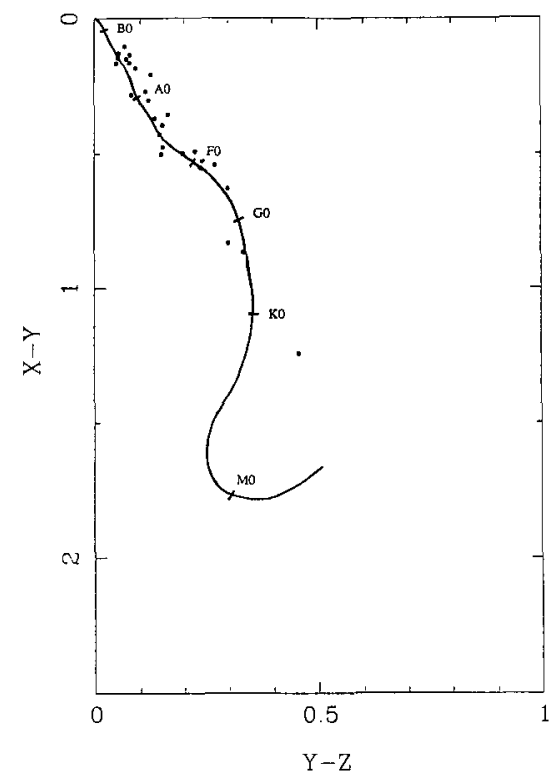

Figure 8: CCD for $O$ Vel galactic cluster with superimposed ZAMS. 


\section{Southern Hemisphere Secondary Standards}

In all some 225 stars have been observed so far in setting up a southern hemisphere network of secondary standards. Figure 4 shows the distribution of stars observed near the south celestial pole and figure 5 those between declinations $-20^{\circ}$ and $-70^{\circ}$. The stars range in magnitude from $\mathrm{V}=1.90$ to 6.90 with spectral types from $\mathrm{O} 5$ to M3 and luminosity classes Ia to V. Figure 6 shows a two dimensional plot of numbers of stars in particular magnitude and spectral ranges. The next phase of the southern hemisphere standards programme will be to measure the magnitudes and colours of selected E-region stars.

\section{Star Cluster Programme}

To date observations have been made of 8 stars in M41, 39 in Kappa Crucis and 43 in IC2391. Figure 7 shows the CM diagram (V vs $(\mathrm{Y}-\mathrm{V})$ ) for the nearby, young cluster $O$ Velorum (IC2391) so far obtained and figure 8 shows a $(\mathrm{X}-\mathrm{Y}),(\mathrm{Y}-\mathrm{Z}) \mathrm{CC}$ plot for the same cluster. A ZAMS is superimposed on the CC diagram. More observations using both photoelectric and CCD photometry have yet to be made of these and other galactic clusters.

\section{Acknowledgements}

We wish to thank the Foundation for Research, Science and Technology and the V.U.W. Internal Research Committee in New Zealand for partial funding of the project; the Physics and Astronomy Department of the University of Canterbury for generous allocation of telescope time at Mt. John Observatory; and the Institute of Theoretical Physics and Astronomy, Vilnius, Lithuania for encouragement and help.

\section{References:}

Hardie R.H., 1962, Photoelectric reductions. In Astronomical Techniques, W. A. Hiltner, editor, volume 2 of Stars and Stellar Systems. The University of Chicago Press, Chicago, Illinois, U.S.A.

Straižys V., 1992, Multicolor Stellar Photometry, volume 15 of Astronomy and Astrophysics Series. Pachart Publishing House, 1130 San Lucas Circle, P.O. Box 35549, Tucson, Arizona 85740, 1992.

Gunn, J.E. and Stryker, L.L., 1983, Astrophys. J. Suppl. 52, 121. 


\section{Discussion}

W. Wisniewski: How do you handle extinction? Vilnius filters are narrow and there are seven of them. With longer exposures between the first and last filter, there will be a large airmass difference.

Sullivan: UPXYZVS observations are made of extinction stars approximately hourly. For the longest integrations (100s), the time required for a full set of observations is approximately 12 minutes. Unless the star is at a large zenith distance, the change in airmass through a single observation is small but in any case, it is allowed for by correcting each colour integration separately for extinction.

W. Tobin: Are the stars you're observing standards in other photometric systems, so that you know that they aren't variable?

Sullivan: Generally the standards so far observed are taken from the Bright Star Catalogue and have been observed in other photometric systems. We deliberately avoided those stars in the Catalogue denoted as variables. In addition, we cross-checked the selected stars using the SIMBAD data base.

R.F. Garrison: It would make transformations between systems a lot easier if you could use the $E$ region standards as your standards. If the Vilnius system can be used to observe $17^{\text {th }}$ magnitude stars with a $1.5 \mathrm{~m}$ telescope, it should be no problem to reach $10^{\text {th }}$ magnitude in the E regions! Why set up a whole new and different system of stars?

Sullivan: We agree and will begin measuring E region standards later this year. We felt that in order to get the programme underway we should start with a selection of bright stars, given the vagaries of the New Zealand weather and our likely access to telescopes of a given aperture.

V. Straižys: What is the magnitude and spectral type range of the Vilnius system standards in the Southern Hemisphere?

Sullivan: The magnitude range is $1.90<\mathrm{V}<6.90$ with spectral types from 05 to $\mathrm{M} 3$. 